Article

\title{
Selection and Characterization of DNA Aptamers for Egg White Lysozyme
}

\author{
Dinh T. Tran ${ }^{1}$, Kris P. F. Janssen ${ }^{1}$, Jeroen Pollet ${ }^{1}$, Elke Lammertyn ${ }^{2}$, Jozef Anné ${ }^{2}$, \\ Ann Van Schepdael ${ }^{3}$ and Jeroen Lammertyn ${ }^{1, *}$
}

1 BIOSYST-MeBioS, Katholieke Universiteit Leuven, Willem de Croylaan 42, B-3001 Leuven, Belgium; E-Mails: ThiDinh.Tran@biw.kuleuven.be (D.T.T.); Kris.Janssen@biw.kuleuven.be (K.P.F.J.); Jeroen.Pollet@biw.kuleuven.be (J.P.)

2 Laboratory of Bacteriology, Rega Institute, Katholieke Universiteit Leuven, Minderbroedersstraat 10, B-3000 Leuven, Belgium; E-Mails: Elke.Lammertyn@1rd.kuleuven.be (E.L.); Jozef.Anne@rega.kuleuven.be (J.A.)

3 Laboratory for Pharmaceutical Analysis, O\&N II Herestraat 49 - bus 923, Katholieke Universiteit Leuven, B-3000 Leuven, Belgium; E-Mail: Ann.VanSchepdael@pharm.kuleuven.be (A.V.S.)

* Author to whom correspondence should be addressed; E-Mail: jeroen.lammertyn@biw.kuleuven.be; Tel.: + 32-16-321459; Fax: + 32-16-321614.

Received: 13 January 2010; in revised form: 10 February 2010 / Accepted: 1 March 2010 / Published: 2 March 2010

\begin{abstract}
We have selected aptamers binding to lysozyme from a DNA library using capillary electrophoresis-systematic evolution of ligands by exponential enrichment. During the selection process the dissociation constant of the ssDNA pool decreased from the micromolar to the low nanomolar range within five rounds of selection. The final aptamer had a dissociation constant of $2.8 \pm 0.3 \mathrm{nM}, 6.1 \pm 0.5 \mathrm{nM}$, and $52.9 \pm 9.1 \mathrm{nM}$ as determined by fluorescence anisotropy, surface plasmon resonance and affinity capillary electrophoresis respectively. The aptamers were successfully challenged for specificity against other egg white proteins. The high affinity aptamers open up possibilities for the development of aptamer based food and medical diagnostics.
\end{abstract}

Keywords: aptamer; capillary electrophoresis; food allergen; lysozyme; systematic evolution of ligands by exponential enrichment 


\section{Introduction}

Lysozyme from hen egg white has been used not only as an anti-inflammatory drug in the treatment of wounds and infections but also as a natural antibacterial agent in food preservation due to its muraminidase activity [1]. Commercially, lysozyme is most often used in cheese production for the prevention of late gas blowing that is caused by the growth of Clostridium tyrobutyricum [2,3]. In the field of enology, it is used to prevent the color loss of red wine [4] and reduce the lactic bacteria flora in musts and wines after completion of malolactic fermentation [5]. Lysozyme has also been added in surimi products, shrimp, and sausage to prolong the shelf-life of these products [6-8].

Besides its useful properties, lysozyme also has its drawbacks. Clinical studies revealed that lysozyme is a dominant egg-white allergen named Gal d 4 [9]. Lysozyme can cause allergic reactions even when present in minute amounts [10-12]. Hence it is of importance to design highly sensitive bioreceptors for detection of this substance in products intended for consumption by allergic patients.

Currently, enzyme-linked immunosorbent assay (ELISA) using antibody for quantification of lysozyme is commercially available. The minimal sensitivity of this assay is $0.021 \mathrm{ng} / \mathrm{mL}$ (Calbiotech Inc, Spring Valley, CA, USA). However, the common disadvantages of ELISA are the complicated succession of steps, long reaction time and the expensive reagents involved.

In recent years, there is an increase in the use of aptamers as biorecognition molecules for biosensors. As bioreceptors, aptamers have distinct advantages over antibodies, such as elimination of batch to batch variability, low cost of manufacturing, and resistance to denaturation and degradation [13]. Aptamers are selected from synthetic nucleic acid libraries using the iterative SELEX (Systematic Evolution of Ligands by EXponential enrichment) process for a wide range of targets such as proteins [14-16], peptides [17], amino acid [18], and organic dyes [19]. There have been a number of reviews describing SELEX in detail [20-22]. Cox and Ellington [23] have selected aptamers for lysozyme using automated selection. Briefly a typical round of selection involved in incubation of the RNA pool with lysozyme captured on magnetic beads, filtration to separate bound sequences from unbound species, and amplification to generate an enriched RNA pool for the next round. After the final round, they obtained an aptamer with a dissociation constant $\left(K_{d}\right)$ of $31 \mathrm{nM}$. This aptamer was further optimized by Kirby et al. [24] to accommodate both immobilization and sensing. Subsequently, the modified aptamer was used as a receptor molecule to develop biosensors for lysozyme detection based on different transduction mechanisms [25-30].

In this paper, we present the selection of DNA aptamers for lysozyme with high affinity and selectivity using capillary electrophoresis-SELEX (CE-SELEX). The main advantages of CE-SELEX over the conventional SELEX are that this method provides very strong power of separation, selections are carried out in solution, thus reducing the non-specific binding introduced by stationary support and it is possible to monitor the evolution of the overall affinity increase of selected aptamers online [31].

The objective of this study was to select DNA aptamers for lysozyme with high affinity and selectivity using CE-SELEX. The selected aptamers were characterized for binding affinity using fluorescence anisotropy, affinity capillary electrophoresis and surface plasmon resonance and finally challenged with other egg white proteins to assess specificity. The selected aptamers with nanomolar dissociation constants may function as biorecognition molecules to develop biosensors for the 
detection and quantification of lysozyme in food and pharmaceuticals to ensure compliance with allergen labeling and improve consumer protection.

\section{Results and Discussion}

\subsection{Selection of aptamers}

CE-SELEX was used to select aptamers against egg-white lysozyme. In order to determine the aptamer collection window, the ssDNA library was incubated with the target. After equilibrium was reached, the mixture was injected into the capillary. Since the electrophoresis mobility of a solute in the capillary depends linearly on its charge to mass ratio, the complex migrated slower than the free DNA when reverse polarity is applied over the capillary. Figure 1 shows that the free DNA reached the detector after $3 \mathrm{~min}$ while the peak of the complex appeared after $4.2 \mathrm{~min}$. To avoid contamination by unbound DNA during sample collection, the collection window was chosen after 3.4 min of separation.

Figure 1. Choosing an aptamer collection window. The injection mixture contained $20 \mathrm{nM}$ fluorescently labeled DNA and $1600 \mathrm{nM}$ lysozyme. CE conditions: TGK buffer, $\mathrm{pH}=8.3$; $1 \mathrm{psi} / 5 \mathrm{~s}$ injection; $25^{\circ} \mathrm{C}$; LIF detection; 40.2-cm, 50- $\mu$ m-i.d. capillary; 20-kV separation voltage. $50 \mathrm{nM}$ BCECF was used as internal standard (IS). The cutoff point where collection of binding sequences is started is marked on the electropherogram by the arrow.

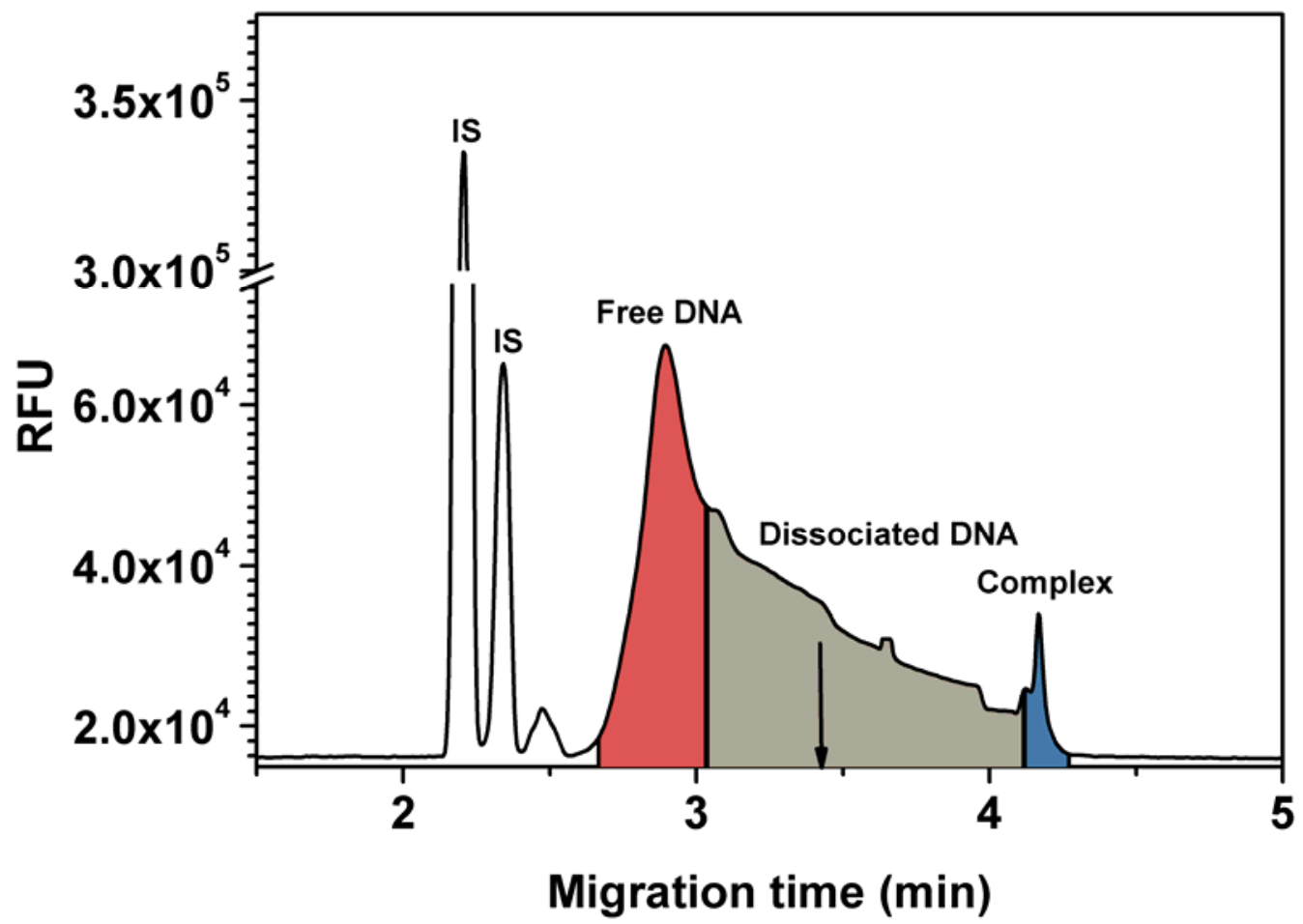

The improvement in binding of the DNA pool with lysozyme after each round of selection was initially evaluated using affinity capillary electrophoresis (ACE). The results in Figure 2 reveal that the average affinity of the DNA pool for the target increased significantly in the first three rounds of 
selection. This is represented by a decrease in free DNA. In round 5, the estimated affinity leveled off, at least two orders of magnitude below the bulk affinity of the naive DNA library. In total, the selection of aptamers for lysozyme required only five selection rounds. This clearly shows the advantages CE-SELEX can hold over traditional SELEX which normally requires anywhere between eight to 15 rounds to obtain aptamers with dissociation constant values in the nanomolar range [32].

Sequence alignment using the ClustalX software program revealed that there were five clones remaining in the enriched DNA pool obtained after the final round. Their sequences are given in Table 1. None of these aptamers has a sequence region which is identical to that of aptamers selected for lysozyme in the study conducted by Cox and Ellington [23] using automated selection. Three clones namely Apta1, Apta3 and Apta8 were selected, based on their abundance, and synthesized for further characterization with respect to affinity and specificity.

Figure 2. Affinity of the DNA library (first bar) and the enriched DNA pools to lysozyme after each CE-SELEX round. Free DNA peak areas were measured by affinity capillary electrophoresis. Error bars indicate the 95\% confidence intervals as determined from nonlinear least-squares regression analysis.

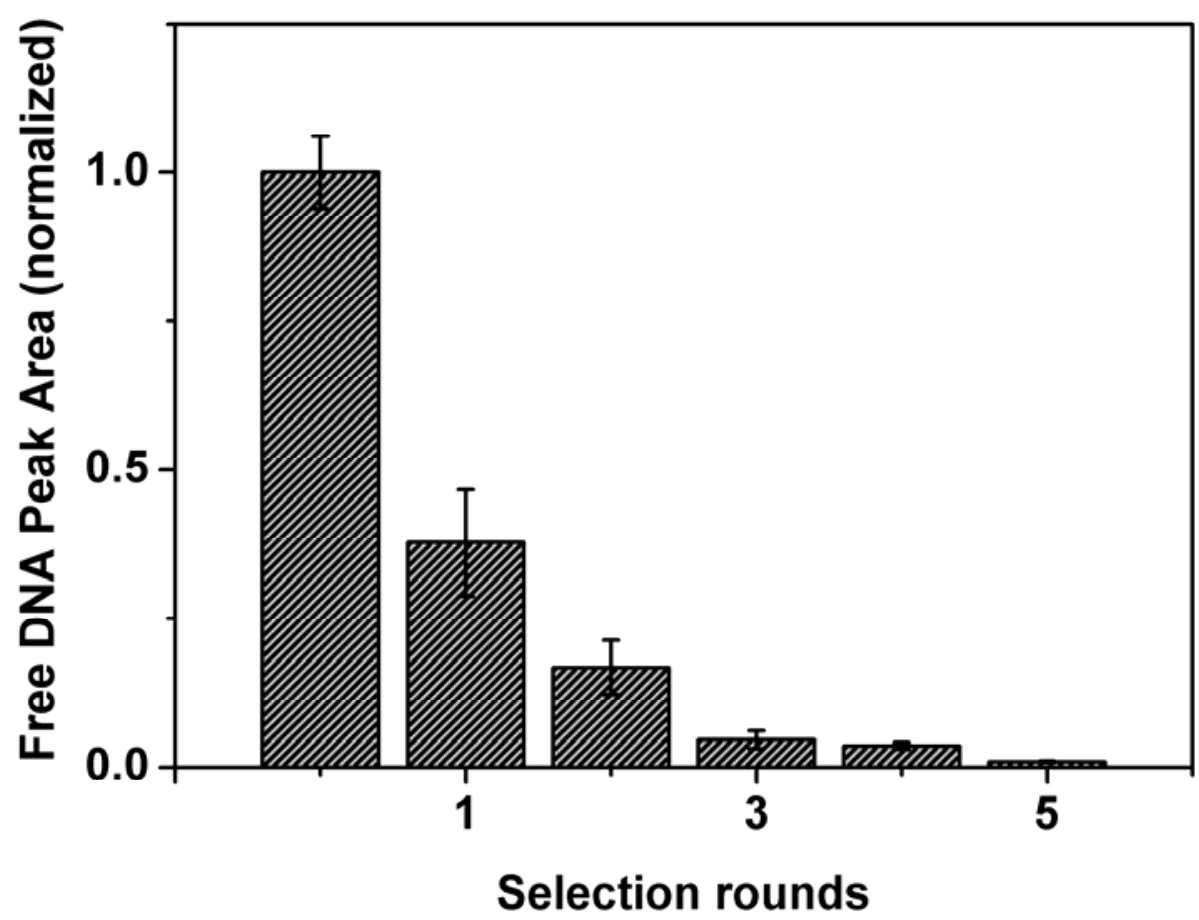

Table 1. Sequences of the clones remaining after five rounds of selection using CESELEX. Only the random region (40 nucleotides) is given.

\begin{tabular}{ll}
\hline Clone ID & \multicolumn{1}{c}{ Sequence of random region (5’ to 3') } \\
\hline Apta1 & GCAGCTAAGCAGGCGGCTCACAAAACCATTCGCATGCGGC \\
Apta2 & GCGTGGGCAGCTAGCACCGATGGTTCTATCGTGGGCTCCG \\
Apta3 & GCGGGTCGGTTGCTCGCTTCGCCCGATCGGTCTAAGGGTG \\
Apta4 & GCGCAAGGTCATCGCATCGCGTCGGAATGGGCTACAGGTG \\
Apta8 & GCACCTTGATGACATGATAGTCGTTGTGTATGCAGTTGGC \\
\hline
\end{tabular}




\subsection{Evaluation of the aptamer binding affinity}

The binding affinity of the aptamers was firstly determined using ACE. All three clones showed high affinity to lysozyme and had very similar $K_{d}$ values when measured using ACE $(52.9 \pm 9.1 \mathrm{nM}, 47.1 \pm 16.3 \mathrm{nM}, 52.1 \pm 12.7 \mathrm{nM}$ for Apta1, Apta3 and Apta8 respectively). This illustrates that clones with different sequences in the final pool have a comparable affinity for lysozyme. Analyses of the secondary structure of the three aptamers using the mfold program showed that the primer regions of Apta1 were in an exterior loop (Figure 3) while the reverse primer of Apta3 was arranged in the stem-loop structure. The free energy of Aptal was also significantly lower than that of Apta3 and Apta8. Therefore, only Apta1 was used in fluorescence anisotropy (FA) and surface plasmon resonance (SPR) for binding study.

Figure 3. Secondary structure prediction of Apta1. The structure was determined using the mfold tool.

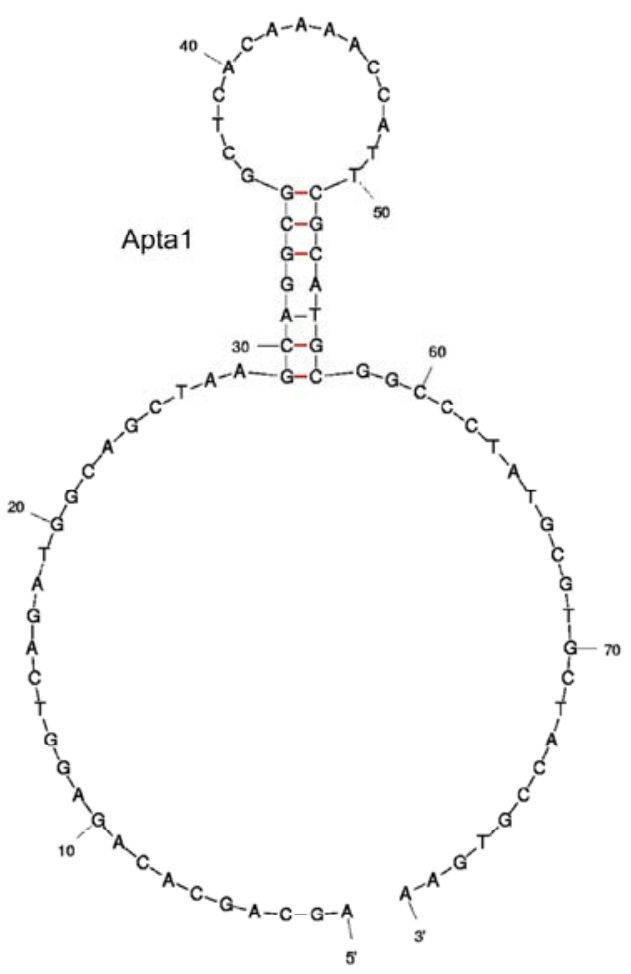

Figure 4 shows the binding curve of Aptal to lysozyme measured by FA. The $K_{d}$ for Apta1 was estimated to be $2.8 \pm 0.3 \mathrm{nM}$. This $K_{d}$ value is about 17 times lower than that obtained using ACE. This is explained by the fact that under the high voltage applied during CE separation, the equilibrium of the mixture was no longer maintained. As a consequence, the complex constantly dissociated which caused smearing of the unbound DNA region (grey area in Figure 1). A part of the complex, which was still intact, generated a single peak (blue area). The decay of the complex made it difficult to determine the end point of the free DNA peak when integrating. In our study, the peak area of the free DNA is shown in the red region in Figure 1 . The $K_{d}$ value obtained by ACE was higher than that obtained by FA which means that we integrated not only the peak of free DNA but also a part of DNA 
which dissociated from the complex. So, FA is a more accurate technique to determine the affinity of the aptamer to lysozyme.

Figure 4. Binding curves of aptamer (Apta1) to lysozyme measured by fluorescence anisotropy. $60 \mu \mathrm{L}$ of $20 \mathrm{nM}$ ssDNA was incubated with the same volume of different concentrations of lysozyme $(0,3.8,302,513$ and $800 \mathrm{nM})$ in quartz cuvettes. The dissociation constant $\left(K_{d}\right)$ was estimated using non-linear least-squares regression analysis. Full line represents the fitted data and symbols represent the measured data given as averages of three measurements. Error bars indicate the $95 \%$ confidence intervals.

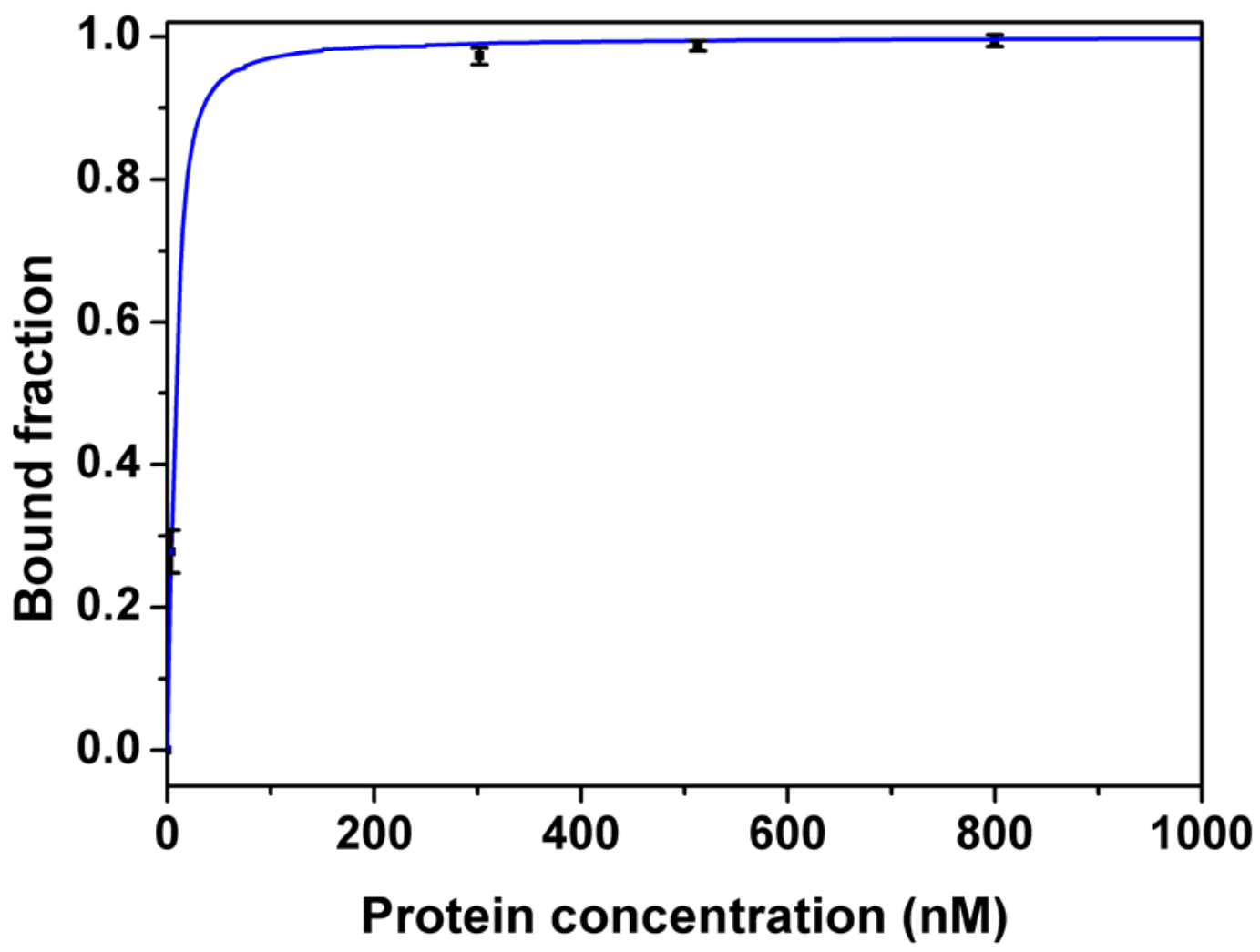

In the SPR measurement, Apta1 was biotinylated and immobilized onto a streptavidin coated SPRchip. In order to minimize mass diffusion effects, the density of the receptor layer was kept to $700 \mathrm{pg}$ of aptamers per $\mathrm{mm}^{2}$ (700 RU). Figure 5 illustrates the high correspondence of the model to the measured kinetic data. Based on this model we estimated the association rate constant, $k_{\text {on }}=6.99 \times 10^{3} \pm 0.04 \times 10^{3} \mathrm{M}^{-1} \mathrm{~s}^{-1}$ and the dissociation rate constant, $k_{\text {off }}=4.3 \times 10^{-5} \pm 0.3 \times 10^{-1} \mathrm{~s}^{-1}$, and hence a $K_{d}$ of $6.1 \pm 0.5 \mathrm{nM}$, confirming the results of the affinity study with fluorescence anisotropy.

However, some caution should be taken when interpreting these SPR results, since we observed limited non-specific interactions between the lysozyme and the bare streptavidin chip. This might be a result of electrostatic interactions between the protein and the sensor surface and hydrophobic interactions between the streptavidin and the lysozyme. As reported previously, these electrostatic interactions can also play an important role in the formation of the complex by a of 'linger and lock' mechanism [33]. We conclude that although the value of the $K_{d}$ is very similar to the results obtained 
in the FA experiments, the kinetic behavior of an lysozyme aptamer bound at a surface might be slightly different compared to the behavior of an unbound aptamer in solution.

Figure 5. Global association/dissociation analysis of the kinetic SPR data on the binding interaction of lysozyme and Apta1. Biotinylated aptamers $(0.5 \mu \mathrm{M})$ were immobilized on a sensor chips and the protein solutions $(200,400,600 \mathrm{nM})$ were injected over the surface.

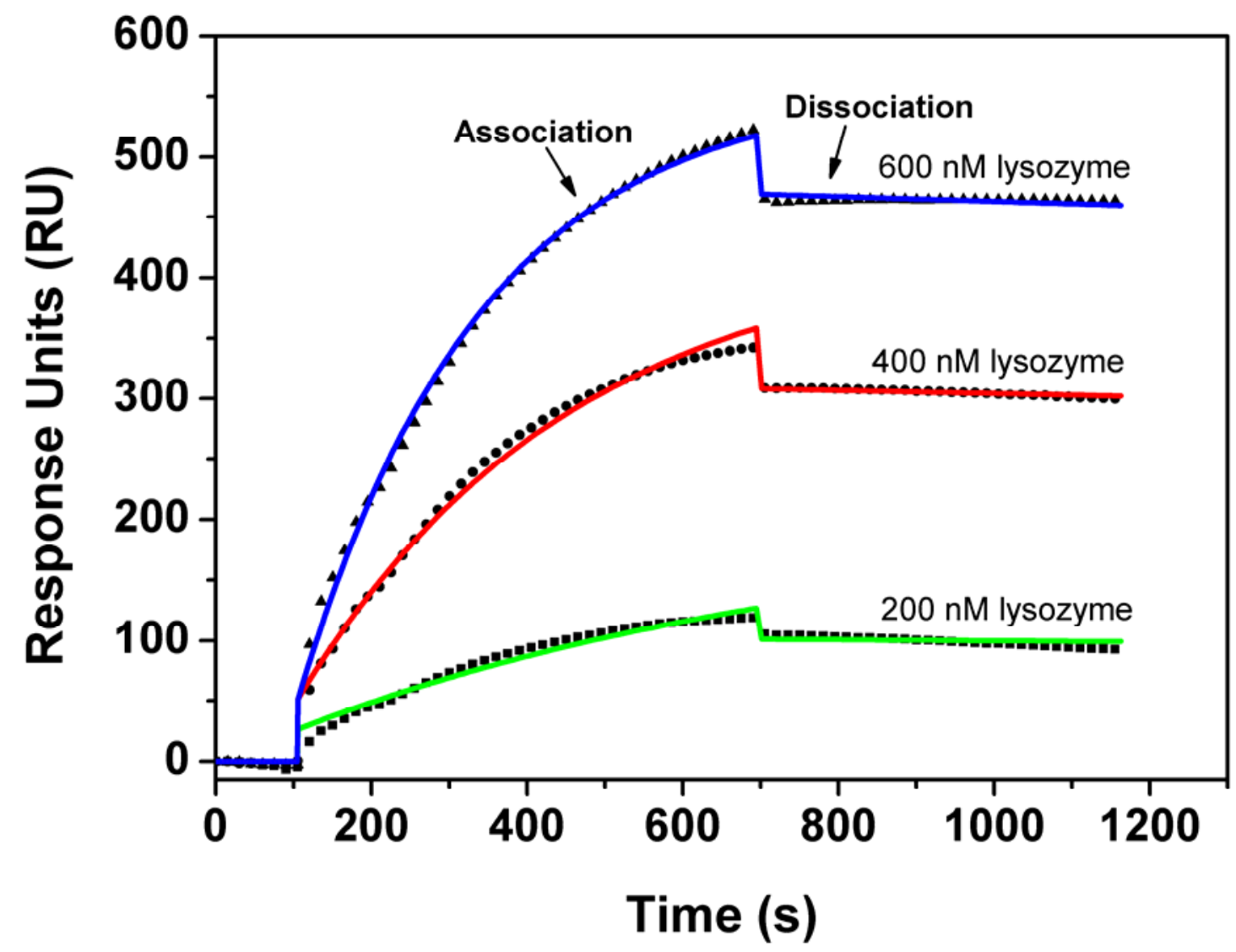

\subsection{Evaluation of binding specificity}

In order to examine the specificity of the selected aptamers to lysozyme, the binding assays were performed using ACE with ovomucoid and ovotransferrin, two proteins causing allergic reactions in egg white, and BSA which is normally used to block the non-specific binding of aptamers to a transducer platform in biosensor applications. Figure 6 indicates that the affinity of the clones Apta1, Apta3 and Apta8 for ovomucoid is orders of magnitude lower than for lysozyme. Although a very small amount of complex was found when checking the binding affinity of the clones to ovomucoid, care should be taken when interpreting these results. According to Ebbehoj et al. [34] commercial ovomucoid is often contaminated with lysozyme, hence, the bound fraction observed might be due to the binding of DNA to lysozyme but not to ovomucoid. For BSA, the affinities of the three clones were determined to be about 5 orders of magnitude lower than the affinity values for lysozyme. These results illustrate that the selected aptamers not only bind strongly to lysozyme but are also very specific and do not bind to other egg white allergenic proteins. 
Figure 6. Specificity of the selected aptamers Apta1, Apta3 and Apta8 for lysozyme and for ovomucoid, ovotransferrin and BSA measured with affinity capillary electrophoresis.

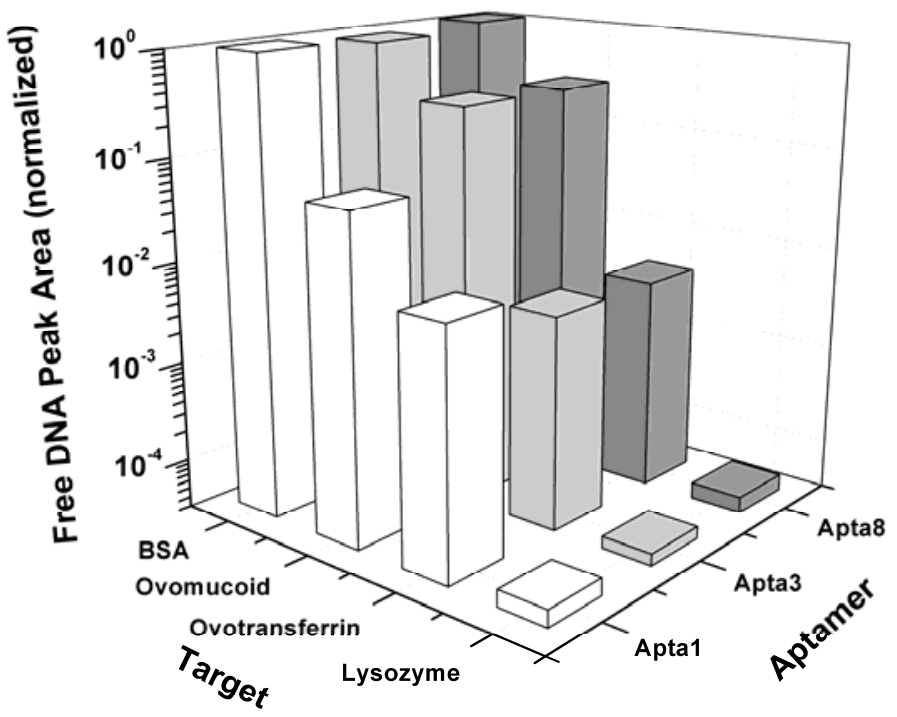

\section{Experimental}

\subsection{Reagents}

Trizma base, lysozyme, ovomucoid and ovotransferrin from chicken egg white, were purchased from Sigma-Aldrich (St. Louis, MO, USA), potassium phosphate dibasic, glycine, sodium hydroxide and EDTA were purchased from ACROS (Fair Lawn, NJ, USA). 2',7'-bis-(2-carboxyethyl)-5-(and-6)carboxyfluorescein (BCECF) was purchased from Invitrogen (Paisley, UK). 'Small fragments' agarose, the DNA library, PCR primers and PCR reagents were purchased from Eurogentec (Liège, Belgium) except for TAQ polymerase and PCR reaction buffer that were purchased from Sphaero-q (Gorinchem, The Netherlands). The DNA library contained 20 nucleotides in the primer regions and 40 nucleotides in a central randomized region (5'-AGC AGC ACA GAG GTC AGA TG - N40 - CCT ATG CGT GCT ACC GTG AA-3'). A 6-carboxyfluorescein (6-FAM) was chemically attached to the 5 ' end of a DNA sequence. The electrophoresis buffer consisted of $25 \mathrm{mM}$ trizma base, $192 \mathrm{mM}$ glycine, and $5 \mathrm{mM} \mathrm{K}_{2} \mathrm{HPO}_{4}$ (TGK) at a $\mathrm{pH}$ of 8.3. Buffer solutions were prepared using HPLC grade water (VWR, Fontenay sous Bois, France) and filtered through a $0.22 \mu \mathrm{m}$ filter (Millipore, Bedford, MA, USA).

\subsection{Capillary electrophoresis operational parameters}

All CE experiments were performed using a P/ACE MDQ capillary electrophoresis system combined with a laser-induced fluorescence (LIF) detector (Beckman Coulter, Inc., Fullerton, CA, USA). As an excitation source a $3 \mathrm{~mW}$ argon ion laser with a wavelength of $488 \mathrm{~nm}$ was used and emission was monitored using a 520/10 nm band pass filter. For all separations a poly(vinyl alcohol) (PVA)-coated capillary (Beckman Coulter, Inc.) with $50 \mu \mathrm{m}$ inner diameter, $360 \mu \mathrm{m}$ outer diameter was used. The total capillary length was $40.2 \mathrm{~cm}$ with a length to detector of $30 \mathrm{~cm}$. The coating of the 
inner wall is intended to decrease non-specific binding of the target to the capillary wall. At the beginning of each experiment, the capillary was rinsed with de-ionized water for $30 \mathrm{~min}$, followed by a rinse with electrophoresis buffer for an additional $15 \mathrm{~min}$. Between injections, the column was rinsed with de-ionized water for $3 \mathrm{~min}$ and with running buffer for $2 \mathrm{~min}$. The temperature of the capillary was maintained at $25{ }^{\circ} \mathrm{C}$ for all separation experiments. Electrophoresis data were collected and analyzed using a 32Karat software, version 8.0 (Beckman Coulter, Inc.).

\subsection{Aptamer selection protocol}

The DNA library and the amplified ssDNA pool after each round of selection were dissolved in TGK electrophoresis buffer, heated to $75^{\circ} \mathrm{C}$ for $10 \mathrm{~min}$ and allowed to cool down on ice. The heating and cooling steps are intended to minimize any misfolding of the aptamers prior to incubation with the protein target. In the first round of selection, $5 \mu \mathrm{L}$ of a $500 \mu \mathrm{M}$ solution of the DNA pool was mixed with $5 \mu \mathrm{L}$ of $200 \mathrm{nM}$ lysozyme and the mixture was incubated for $30 \mathrm{~min}$ at room temperature before electrophoretic separation. In the following rounds, the enriched DNA pools were incubated with decreasing lysozyme concentrations; $50 \mathrm{nM}$ in round $2,25 \mathrm{nM}$, in round 3, $12.5 \mathrm{nM}$ in round 4 and round 5 under conditions identical to those described above. This was done to increase the stringency of the overall selection process.

After incubation, a plug of equilibrium mixture was hydrodynamically injected into the capillary by applying a pressure of 5 psi for $10 \mathrm{~s}$, which corresponds to an injection volume of approximately $130 \mathrm{~nL}$. Separation was carried out at a constant voltage of $20 \mathrm{kV}$ for $4.5 \mathrm{~min}$ with reverse polarity. During this time any unbound species were allowed to elute from the column into a vial of run buffer that was later discarded. When the complexed species reached the end of the column, the target vial was switched and the binding sequences remaining on the capillary were flushed into a collection vial containing $30 \mu \mathrm{L}$ of water by pressure rinsing of the column of $10 \mathrm{psi}$ for $1 \mathrm{~min}$. The exact time at which the complex reaches the end of the capillary was calculated by correcting the complex detection time for the distance between the detection window and the capillary end according to the factor

$f=L_{\text {total }} / L_{\text {detector }}$. Two repeated separations were carried out for each selection round to increase the number of collected sequences.

\subsection{PCR and dsDNA separation}

After separation by $\mathrm{CE}$, the collected volume containing active sequence was amplified using the polymerase chain reaction (PCR) protocol described by Mendonsa and Bowser [35]. Briefly, a $30 \mu \mathrm{L}$ volume of the collected solution was divided into 6 volumes of $5 \mu \mathrm{L}$ each. PCR master mix was added to give a final volume of $50 \mu \mathrm{L}$. All vials were then transferred into a thermal cycler (PerkinElmer, Massachusetts, USA) and heated to $95^{\circ} \mathrm{C}$ for $5 \mathrm{~min}$ to denature the DNA-lysozyme complex. Then the PCR cycling was started. A total of 18 cycles was performed in this way. The size and purity of PCR products was subsequently checked by running samples in $2.5 \%$ agarose gel for $30 \mathrm{~min}$ at $140 \mathrm{~V}$ followed by ethidium bromide-staining.

After amplification, the product was purified using PCR clean-up kit (NucleoSpin, Düren, Germany) to remove any residual primers. The obtained dsDNA sequences were then separated into single stranded form using streptavidin functionalized magnetic beads (Invitrogen, Oslo, Norway). 
A $20 \mu \mathrm{L}$ suspension of the beads was washed three times with $100 \mu \mathrm{L}$ single concentration binding buffer (10 $\mathrm{mM}$ trizma base, $50 \mathrm{mM} \mathrm{NaCl}$, and $1 \mathrm{mM}$ EDTA $\mathrm{pH}=7.5)$. The cleaned beads were then re-suspended in $50 \mu \mathrm{L}$ double concentration binding buffer. Fifty $\mu \mathrm{L}$ of purified dsDNA was added in the mixture and was allowed to react at room temperature for 1 hour under gentle shaking to ensure all dsDNA was properly bound to the magnetic beads. The beads were then washed three times with $100 \mu \mathrm{L}$ single concentration binding buffer. The beads were re-suspended once more in $15 \mu \mathrm{L}$ of $0.2 \mathrm{M} \mathrm{NaOH}$ and left for $5 \mathrm{~min}$ under continuous shaking at room temperature to release the desired ssDNA from its complement that remained bound to the magnetic beads which were removed from the solution. Finally, the ssDNA was precipitated by bringing $15 \mu \mathrm{L}$ DNA solution into a microcentrifuge tube containing $45 \mu \mathrm{L}$ absolute ethanol and $1.5 \mu \mathrm{L}$ of $3 \mathrm{M} \mathrm{CH}_{3} \mathrm{COONa}$ at $\mathrm{pH}$ 5.5. Samples were incubated at $-20{ }^{\circ} \mathrm{C}$ overnight. The precipitated ssDNA was separated from the supernatant by centrifugation at $20.000 \mathrm{~g}$ for $60 \mathrm{~min}$ at $4{ }^{\circ} \mathrm{C}$. Subsequently, the supernatant was decanted from the sample tube. The ssDNA pellet was allowed to dry at $65{ }^{\circ} \mathrm{C}$ for $5 \mathrm{~min}$. The dried pellet was then re-suspended in electrophoresis buffer to serve as a new enriched ssDNA pool for further selection rounds.

\subsection{Cloning and sequencing}

The DNA pool obtained after the fifth round of selection was subjected to PCR amplification with non-labeled primers. The conditions were modified to accommodate further cloning and sequencing of the obtained DNA pool. The PCR products were further purified using a gel extraction kit (NucleoSpin, Düren, Germany). The eluted ssDNA was cloned into the pGEM-T easy vectors (Promega, Madison, WI, USA). High efficiency, E. coli competent cells $\left(\geq 1 \times 10^{8} \mathrm{cfu} / \mu \mathrm{g}\right.$ DNA) (Promega, Madison, USA) were transformed using the vectors containing DNA fragments of interest. The successful transformation was verified by PCR of the resulting bacterial colonies using nonlabeled primers to amplify the inserts and followed by electrophoresis on a $2.5 \%$ agarose gel. Fourteen colonies were then chosen at random for sequencing using the M13 forward primer (Genetic Service Facility, University of Antwerp, Belgium). The secondary structure of the selected aptamers was analyzed using mfold program [36].

\subsection{Binding affinity}

\subsubsection{Affinity capillary electrophoresis}

In order to check the progress of aptamer selection and to assess the binding affinity of the aptamer pool, an average affinity value - Expressed as peak area of free DNA — was determined after every round of selection with CE. For this purpose, a $20 \mathrm{nM}$ sample of ssDNA was pre-incubated with increasing amounts of lysozyme in the presence of BCECF as internal standard (IS). After 30 min of incubation at room temperature the mixture was separated electrophoretically under conditions identical to those used for aptamer selection except that the samples were injected under the pressure of 1 psi for 5s. The peak areas of the relevant species on the resulting electropherograms were determined by integration. The model presented by Tetin and Hazlett [37] was applied to determine the 
$K_{d}$ of aptamers using the non-linear least-squares regression analysis performed with statistical analysis system (SAS) (SAS Institute, Inc., Cary, NC, USA).

\subsubsection{Fluorescence anisotropy}

FA measurements were performed using a SPEX FluoroLog 3 Fluorometer (Horiba Jobin Yvon Inc., Edison, NJ, USA). In this assay, $60 \mu \mathrm{L}$ of $20 \mathrm{nM}$ ssDNA was incubated with the same volume of different concentrations of lysozyme $(0,3.8,302,513$ and $800 \mathrm{nM})$ in quartz cuvettes (Hellma, Germany). The fluorescein-labeled conjugates were excited at $472 \mathrm{~nm}$ and fluorescence was collected at $512 \mathrm{~nm}$. The anisotropy values were used to estimate $K_{d}$ using the same model structure as applied for ACE.

\subsubsection{Surface plasmon resonance}

SPR experiments were performed on a Biacore 3000 (Biacore, GE Healthcare, Uppsala, Sweden). Biotinylated aptamers $(0.5 \mu \mathrm{M}$ in $10 \mathrm{mM}$ Tris- $\mathrm{HCl}, 300 \mathrm{mM} \mathrm{NaCl}$, and $1 \mathrm{mM}$ EDTA at $\mathrm{pH}=7.5)$ were immobilized on streptavidin coated SA-sensor chips (Biacore). The validated aptamers were extended with 24 thymidine bases at the 3 ' biotin binding site, to give them maximum flexibility for binding with the target [38]. Conforming to the ACE and the FA experiments, the kinetic interaction between the aptamer and lysozyme was studied in TGK buffer. The regeneration of the aptamer coated surface was achieved with a $30 \mathrm{~s}$ pulse of a $10 \mathrm{mM}$ glycine $/ \mathrm{HCl}$ buffer $(\mathrm{pH}=2.5)$. The statistical analysis of the kinetic data of the SPR measurements was done based on a 1:1 Langmuir model using Prism (Graphpad Software, San Diego, CA, USA).

\section{Conclusions}

We have successfully selected DNA aptamers with a strong affinity and selectivity towards lysozyme using capillary electrophoresis. This technique allows monitoring of the relative affinity increase of the aptamer pool during the SELEX procedure, but is less accurate for the absolute estimation of the dissociation constant due to a poor peak resolution. Following selection the affinity constants for the complexation reaction of selected aptamers with lysozyme were accurately determined using fluorescence anisotropy and resulted in a value of $2.8 \mathrm{nM}$. SPR measurements confirmed these results but also illustrated the tendency of lysozyme for non-specific binding to the sensor surface. The selection and characterization of this high affinity aptamer opens up possibilities for the development of aptamer based food and medical diagnostics.

\section{Acknowledgements}

This research has been supported by the KULeuven (IOF KP/06/011), the Institute for the Promotion of Innovation by Science and Technology in Flanders (IWT-Vlaanderen) and the Fund for Scientific Research Flanders (FWO G.0298.06 and FWO G.0337.08). Engelborghs and Eef Meyen are gratefully acknowledged for their help with the fluorescence anisotropy measurements and the cloning experiments, respectively. 


\section{References}

1. Proctor, V.A.; Cunningham, F.E. The chemistry of lysozyme and its use as a food preservative and a pharmaceutical. Crit. Rev. Food Sci. Nutr. 1988, 26, 359-395.

2. Wasserfall, F.; Teuber, M. Action of egg-white lysozyme on Clostridium tyrobutyricum. Appl. Environ. Microbiol. 1979, 38, 197-199.

3. Masschalck, B.; Michiels, C.W. Antimicrobial properties of lysozyme in relation to foodborne vegetative bacteria. Crit. Rev. Microbiol. 2003, 29, 191-214.

4. Weber, P.; Kratzin, H.; Brockow, K.; Ring, J.; Steinhart, H.; Paschke, A. Lysozyme in wine: A risk evaluation for consumers allergic to hen's egg. Mol. Nutr. Food Res. 2009, 53, 1469-1477.

5. Gerbaux, V.; Villa, A.; Monamy, C.; Bertrand, A. Use of lysozyme to inhibit malolactic fermentation and to stabilize wine after malolactic fermentation. Am. J. Enol. Vitic. 1997, 48, 49-54.

6. Akashi, A.; Oono, A. Preservative effect of egg-white lysozyme to nonpackaged kamaboko. $J$. Agric. Chem. Soc. Jpn. 1972, 46, 177-183.

7. Chander, R.; Lewis, N.F. Effect of lysozyme and sodium EDTA on shrimp microflora. Eur. J. Appl. Microbiol. Biotechnol. 1980, 10, 253-258.

8. Samuelson, K.J.; Rupnow, J.H.; Froning, G.W. The effect of lysozyme and ethylenediaminetetraacetic acid on Salmonella on broiler Parts. Poult. Sci. 1985, 64, 1488-1490.

9. Anet, J.; Back, J.F.; Baker, R.S.; Barnett, D.; Burley, R.W.; Howden, M.E.H. Allergens in the white and yolk of hens egg - a study of IgE binding by egg proteins. Int. Arch. Allergy Appl. Immunol. 1985, 77, 364-371.

10. Perez-Calderon, R.; Gonzalo-Garijo, M.A.; Lamilla-Yerga, A.; Mangas-Santos, R.; MorenoGaston, I. Recurrent angioedema due to lysozyme allergy. J. Invest. Allergol. Clin. Immunol. 2007, 17, 264-266.

11. Yman, I.M. Detection of inadequate labeling and contamination as causes of allergic reactions to food. Acta Aliment. 2004, 33, 347-357.

12. Fremont, S.; Kanny, G.; Nicolas, J.P.; MoneretVautrin, D.A. Prevalence of lysozyme sensitization in an egg-allergic population. Allergy 1997, 52, 224-228.

13. Tombelli, S.; Minunni, A.; Mascini, A. Analytical applications of aptamers. Biosens. Bioelectron. 2005, 20, 2424-2434.

14. Bock, L.C.; Griffin, L.C.; Latham, J.A.; Vermaas, E.H.; Toole, J.J. Selection of single-strandedDNA molecules that bind and inhibit human thrombin. Nature 1992, 355, 564-566.

15. Wen, J.D.; Gray, C.W.; Gray, D.M. SELEX selection of high-affinity oligonucleotides for bacteriophage Ff gene 5 protein. Biochemistry 2001, 40, 9300-9310.

16. Nonaka, Y.; Sode, K.; Ikebukuro, K. Screening and improvement of an anti-VEGF DNA aptamer. Molecules 2010, 15, 215-225.

17. Mendonsa, S.D.; Bowser, M.T. In vitro selection of aptamers with affinity for neuropeptide Y using capillary electrophoresis. J. Am. Chem. Soc. 2005, 127, 9382-9383.

18. Geiger, A.; Burgstaller, P.; vonderEltz, H.; Roeder, A.; Famulok, M. RNA aptamers that bind Larginine with sub-micromolar dissociation constants and high enantioselectivity. Nucleic Acids Res. 1996, 24, 1029-1036. 
19. Ellington, A.D.; Szostak, J.W. Selection Invitro of single-stranded-DNA molecules that fold into specific ligand-binding structures. Nature 1992, 355, 850-852.

20. Osborne, S.E.; Ellington, A.D. Nucleic acid selection and the challenge of combinatorial chemistry. Chem. Rev. 1997, 97, 349-370.

21. Stoltenburg, R.; Reinemann, C.; Strehlitz, B. SELEX-A (r)evolutionary method to generate highaffinity nucleic acid ligands. Biomol. Eng. 2007, 24, 381-403.

22. Pan, W.H.; Clawson, G.A. The shorter the better: reducing fixed primer regions of oligonucleotide libraries for aptamer selection. Molecules 2009, 14, 1353-1369.

23. Cox, J.C.; Ellington, A.D. Automated selection of anti-protein aptamers. Bioorg. Med. Chem. 2001, 9, 2525-2531.

24. Kirby, R.; Cho, E.J.; Gehrke, B.; Bayer, T.; Park, Y.S.; Neikirk, D.P.; McDevitt, J.T.; Ellington, A.D. Anal. Chem. 2004, 76, 4066-4075.

25. Kawde, A.N.; Rodriguez, M.C.; Lee, T.M.H.; Wang, J. Label-free bioelectronic detection of aptamer-protein interactions. Electrochem. Commun. 2005, 7, 537-5340.

26. Rodriguez, M.C.; Kawde, A.N.; Wang, J. Aptamer biosensor for label-free impedance spectroscopy detection of proteins based on recognition-induced switching of the surface charge. Chem. Commun. 2005, 4267-4269.

27. Cheng, A.K.H.; Ge, B.; Yu, H.Z. Aptamer-based biosensors for label-free voltammetric detection of lysozyme. Anal. Chem. 2007, 79, 5158-5164.

28. Huang, H.P.; Jie, G.F.; Cui, R.J.; Zhu, J.J. DNA aptamer-based detection of lysozyme by an electrochemiluminescence assay coupled to quantum dots. Electrochem. Commun. 2009, 11, 816-818.

29. Rodriguez, M.C.; Rivas, G.A. Label-free electrochemical aptasensor for the detection of lysozyme. Talanta 2009, 78, 212-216.

30. Peng, Y.G.; Zhang, D.D.; Li, Y.; Qi, H.L.; Gao, Q.; Zhang, C.X. Label-free and sensitive faradic impedance aptasensor for the determination of lysozyme based on target-induced aptamer displacement. Biosens. Bioelectron. 2009, 25, 94-99.

31. Mendonsa, S.D.; Bowser, M.T. In vitro evolution of functional DNA using capillary electrophoresis. J. Am. Chem. Soc. 2004, 126, 20-21.

32. Luzi, E.; Minunni, M.; Tombelli, S.; Mascini, M. New trends in affinity sensing: aptamers for ligand binding. Trac-Trends Anal. Chem. 2003, 22, 810-818.

33. Law, M.J.; Linde, M.E.; Chambers, E.J.; Oubridge, C.; Katsamba, P.S.; Nilsson, L.; Haworth, I.S.; Laird-Offringa, I.A. The role of positively charged amino acids and electrostatic interactions in the complex of U1A protein and U1 hairpin II RNA. Nucleic Acids Res. 2006, 34, 275-285.

34. Ebbehoj, K.; Dahl, A.M.; Frokiaer, H.; Norgaard, A.; Poulsen, L.K.; Barkholt, V. Purification of Egg-White Allergens. Allergy 1995, 50, 133-141.

35. Mendonsa, S.D.; Bowser, M.T. In vitro selection of high-affinity DNA ligands for human IgE using capillary electrophoresis. Anal. Chem. 2004, 76, 5387-5392.

36. Zuker, M. Mfold web server for nucleic acid folding and hybridization prediction. Nucleic Acids Res. 2003, 31, 3406-3415.

37. Tetin, S.Y.; Hazlett, T.L. Optical spectroscopy in studies of antibody-hapten interactions. Methods 2000, 20, 341-361. 
38. Katilius, E.; Flores, C.; Woodbury, N.W. Exploring the sequence space of a DNA aptamer using microarrays. Nucleic Acids Res. 2007, 35, 7626-7635.

Sample Availability: Samples of the compounds are available from authors.

(C) 2010 by the authors; licensee Molecular Diversity Preservation International, Basel, Switzerland. This article is an open-access article distributed under the terms and conditions of the Creative Commons Attribution license (http://creativecommons.org/licenses/by/3.0/). 